\title{
Thermally activated carrier transfer processes in InGaN/GaN multi-quantum-well light-emitting devices
}

\author{
C. L. Yang, L. Ding, J. N. Wang, K. K. Fung, and W. K. Ge ${ }^{\text {a) }}$ \\ Department of Physics and Institute of Nano Science and Technology, Hong Kong University of Science and \\ Technology, Hong Kong, China \\ H. Liang, L. S. Yu, Y. D. Qi, D. L. Wang, Z. D. Lu, and K. M. Lau \\ Department of Electrical Engineering and Electronics, Hong Kong University of Science and Technology, \\ Hong Kong, China
}

(Received 14 March 2005; accepted 27 May 2005; published online 19 July 2005)

\begin{abstract}
We have studied the temperature-dependent carrier transfer processes in InGaN/GaN multi-quantum-well light-emitting devices using various optical techniques such as photoluminescence, electroluminescence, and photoluminescence excitation spectra. The role of the defects in the $\mathrm{GaN}$ barrier neighboring to the InGaN region was demonstrated clearly in capturing carriers only at low temperatures. The physical origin of the defects was most possibly attributed to the stacking faults at the interface according to the high-resolution transmission electron spectroscopy pictures. (C) 2005 American Institute of Physics. [DOI: 10.1063/1.1978967]
\end{abstract}

\section{INTRODUCTION}

Carrier localization ${ }^{1-4}$ is widely observed in InGaN potential wells and commonly referred as an interpretation for the coexistence of high quantum yield of the light emission as well as high density of threading dislocations. Carriers are localized in the defect-free space $(\mathrm{InGaN}, \mathrm{InN}$, or In-rich quantum dots) as to avoid reaching impurity sites where they are annihilated radiatively or nonradiatively. Therefore, the localization effect helps us to make the radiative recombination process efficient. We may, however, find that this would only promise an efficient radiation once carriers were injected into the quantum dots. As the high-density defects would certainly capture the electrically or optically injected carriers before they can relax to the energetically favorable quantum dots, an ineligible carrier loss channel is available. Here we focus on the transport of the injected carriers in the GaN barriers adjacent to the quantum dots, where they might be captured by either defects or quantum dots. The carrier transfer process thereafter will give us a more detailed understanding on the success of the light-emitting devices (LEDs) with high quantum efficiency. We have, at low temperature, observed the loss channel of the injected carriers as being captured by defects in the GaN barriers nearby the quantum dots, which makes the carriers transfer to the quantum dots less efficient. But the process of carriers release from the defects and transfer into the energetically lowerlying quantum dot states are thermally activated at higher temperature $(>100 \mathrm{~K})$, resulting in an efficient carrier injection into the quantum dots at elevated temperatures.

\section{EXPERIMENTS AND DISCUSSIONS}

Bright InGaN/GaN LEDs consisting of five periods of multiquantum wells were grown by metal-organic chemical-

\footnotetext{
${ }^{\text {a) }}$ Author to whom correspondence should be addressed; electronic mail: phweikun@ust.hk
}

vapor deposition (MOCVD). The indium composition, the well thickness $(2-4 \mathrm{~nm})$, and the barrier width $(8-12 \mathrm{~nm})$ in the quantum wells were tuned to achieve blue, green, and yellow-green light emissions, respectively. The typical output power $(I=20 \mathrm{~mA})$ of the unpackaged blue and green LEDs is about $1.5-2 \mathrm{~mW}$. For the temperature-dependent photoluminescence (PL) and electroluminescence (EL) measurements, the samples were mounted inside a close-cycled $\mathrm{He}$ cryostat. For selectively excited $\mathrm{PL}$, a $\mathrm{He}-\mathrm{Cd}$ laser or the second-harmonic generation of a tunable Ti-sapphire femtosecond laser was used for excitation. In the photoluminescence excitation (PLE) spectra measurement, a light source of a 300-W Xe lamp dispersed by a $0.25-\mathrm{m}$ monochromator was employed for excitation. For all of the spectral measurement, the light emission from the samples was firstly dispersed by a $0.5-\mathrm{m}$ spectrometer and then detected by a photomultiplier tube. The transmission electron microscopy pictures were taken using a JEOL 2010F system to show the microstructure of the samples.

In Fig. 1, the EL of the blue and green LEDs with fixed injection current of $1 \mathrm{~mA}$ at both 11 and $300 \mathrm{~K}$ is presented. At $11 \mathrm{~K}$, the green LED shows the InGaN-related main peak at $2.35 \mathrm{eV}$ [labeled as quantum dot (QD)], accompanied by a higher-energy peak at $3.27 \mathrm{eV}$ (labeled as $D$ peak) with a comparable intensity. When the temperature goes to $300 \mathrm{~K}$, the QD-related peak is intensified and broadened compared with that at $11 \mathrm{~K}$, witnessing the totally disappearing of the $D$ peak. For the blue LED, the general features are the same as those of the green LED except that the $D$ peak was much weaker in the EL spectrum even at low temperatures. The QD peaks for both blue and green diodes coincide with the low-energy asymptotes of the PLE spectra, indicating its origin of localized excitons at the potential minimum, rather than at the peak of the joint density of states. The origin of the unknown higher-energy $D$ peak will be discussed later.

The Arrhenius plot $(1000 / T)$ of the detailed temperature dependence of the integrated EL intensities of the QD peak 


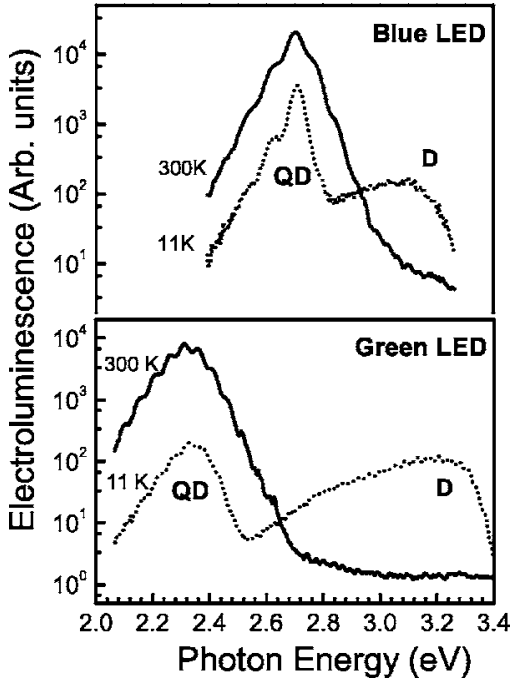

FIG. 1. EL spectra of blue and green LEDs at 11 and $300 \mathrm{~K}$ as indicated.

and $D$ peak is shown in Fig. 2. From 10 to about $100 \mathrm{~K}$, those two peaks both undergo a slow increase as the temperature goes up. But when the temperature goes further to be higher than $100 \mathrm{~K}$, it can clearly be seen that the QDrelated peak intensity experiences a sudden climbing at the expense of a sharp intensity falling of the $D$ peak. The QDpeak intensity starts to decrease only when the temperature is higher than $250 \mathrm{~K}$, which is in contrast with the monotonic decrease of the $D$-peak intensity above $100 \mathrm{~K}$. Obviously, at low temperatures, the $D$-peak-related recombination process as well as some possible nonradiative recombination processes at the defect sites can be taken as an effective carrier loss channel so that the injected carriers cannot be efficiently captured by the InGaN QDs. The carrier transfer process to the QDs is, however, thermally activated above $100 \mathrm{~K}$, resulting in a dramatic increase of the QD emission and a

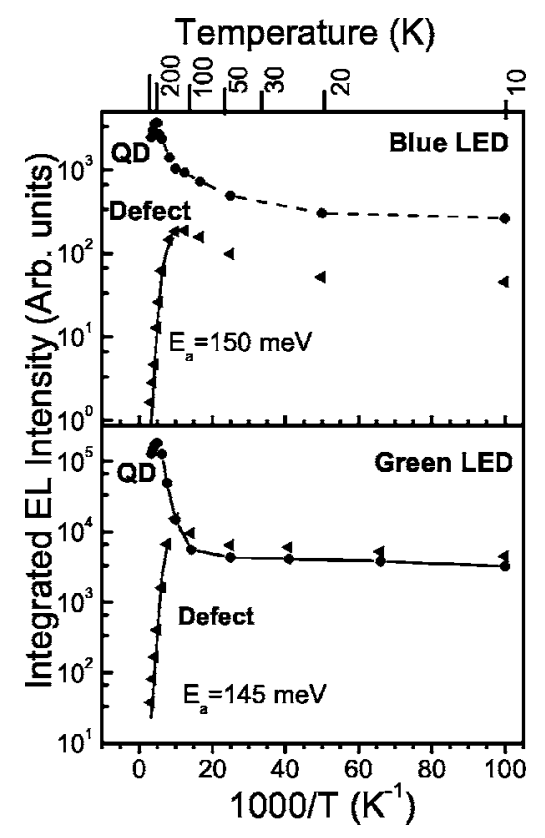

FIG. 2. The temperature dependence of the integrated EL intensities of the QDs peak and the $D$ peak for both green and blue LEDs.

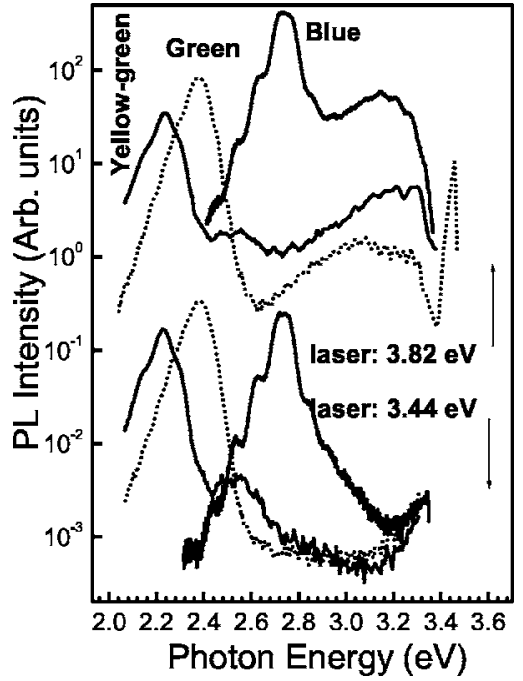

FIG. 3. PL spectra of blue, green, and yellow-green InGaN LEDs excited with laser energy at 3.82 and $3.44 \mathrm{eV}$, respectively.

simultaneous drop of the $D$ peak. We have used the thermal activation process to fit the decrease of the $D$-peak intensity, which gives the thermal activation energy of $E_{a}=150 \mathrm{meV}$ and $E_{a}=145 \mathrm{meV}$ for the blue and green LEDs, respectively.

The double-peak feature and the temperature-dependent behavior in the EL spectra are very common in our blue, green, and yellow-green LED samples. We noted that O'Donnell et al. ${ }^{4}$ have reported similar two peaks from commercial Nichia blue and green diodes in their lowtemperature EL emission. By comparing the EL and photocurrent spectra, they have offered a description of the luminescence origin in the Nichia LEDs as follows: Rather than forming a conventional alloy, the highly restricted solubility of In in GaN (less than 6\% at typical growth temperatures, according to theoretical estimations) was believed to lead to an InGaN active layer composed of quantum dots of nearly uniform composition (approaching $\mathrm{InN}$ ) embedded in an indium-deficient matrix $\left(\operatorname{In}_{x} \mathrm{Ga}_{1-x} \mathrm{~N}\right.$, with $\left.x \sim 0.06\right)$. Increasing the indium incorporation was thought to merely increase the mean size of the quantum dots, while the composition stays pinned. The weaker but higher-energy peak was then attributed to the corresponding transition predicted for a random alloy of InGaN matrix. Hori et al. ${ }^{5,6}$ studied the temperature-dependent EL spectra in efficient blue and green LEDs produced also by Nichia, showing general features very similar to our observation. They had attributed the higher-energy peak possibly to $\mathrm{GaN}$, although a large redshift was noticed compared with the band gap of GaN.

To understand the origin of the $D$ peak, selectively excited PL spectra were measured at $11 \mathrm{~K}$ with excitation laser energy of 3.82 or $3.44 \mathrm{eV}$, respectively, which is just above and below the band-gap energy $(3.504 \mathrm{eV})$ of the $\mathrm{GaN}$ barrier. In Fig. 3 we can find that when the laser is chosen to allow both the $\mathrm{GaN}$ barrier and InGaN QDs excited, the PL spectra of the LEDs show two peaks just as those observed in the EL spectra. But the PL spectra are absolutely clear of the $D$ peak if the laser energy is tuned just below the band gap of the GaN barrier. It is clearly demonstrated that the $D$ peak originated from some kind of defect levels in GaN. The 


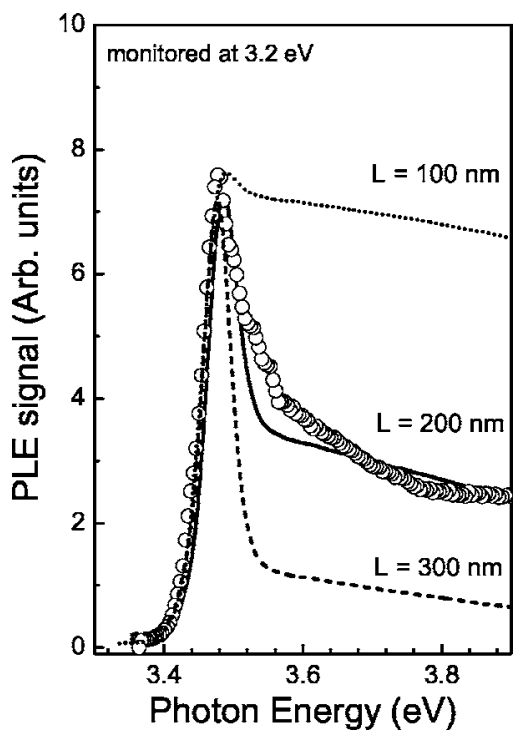

FIG. 4. PLE spectrum (open circle) monitored at the $D$ peak of the green LED at $11 \mathrm{~K}$. The solid curve is the theoretical calculation using parameter $d=200 \mathrm{~nm}$, the same as the sample structure. The dashed and dotted curves are the calculated results using $d=100$ and $300 \mathrm{~nm}$, resulting in symbatic and antibatic spectral responses compared to that of the absorption of $\mathrm{GaN}$.

PLE spectrum of the green LED (the open circle curve in Fig. 4) monitored at $3.2 \mathrm{eV}$ shows a maximum contribution at the band-gap energy of $\mathrm{GaN}$ and then a sharp decrease when the photon energy is smaller. Here we do not see any proof that the high-energy peak is related to the so-called random alloy of InGaN in our sample. By carefully examining the line shape of the PLE spectrum, we can find that the defects must be located at a certain depth below the surface where QDs are embedded, rather than from the GaN capping or buffer layer. If the signal is just coming from a certain region below the surface, then the luminescence intensity can be described by the following equation ${ }^{7,8}$ to include the contribution of both absorption and carrier diffusions:

$$
\begin{aligned}
I= & \frac{\alpha \theta I_{0} D e}{\left(\beta^{2}-\alpha^{2}\right)} \\
& \times \frac{\beta\left(e^{\beta L}-e^{-\alpha L}\right)+\beta\left(e^{-\beta L}-e^{-\alpha L}\right)+\alpha\left(e^{-\beta L}-e^{\beta L}\right)}{e^{-\beta L}-e^{\beta L}},
\end{aligned}
$$

where $\theta$ is the carrier generation efficiency, $\alpha$ the absorption coefficient, ${ }^{9} I_{0}$ the incident light flux, $\beta$ the inverse of the mean carrier transfer length, and $L$ the depth from the surface to the luminescent region. Since $\alpha$ is the function of photon energy, the higher the photon energy, the larger the absorption and thus the less the excitation light penetration into the QD region. Therefore the line shape of the PLE should strongly depend on the depth where the defects are located. The solid line in Fig. 4 is the theoretical curve of the dependence of the luminescence intensity on photon energy, calculated based on Eq. (1) for the PLE signal, taking the experimental parameter $L_{\text {cap }}=200 \mathrm{~nm}$ and mean carrier transfer length of $4 \mathrm{~nm}$ (Ref. 10) ( half of the barrier width). This shows an excellent agreement with the experimental curve. The calculated result using $L=100 \mathrm{~nm}$ (dashed line) and $300 \mathrm{~nm}$ (dotted line) is of totally different line shapes from the experimental results, being either symbatic or antibatic in

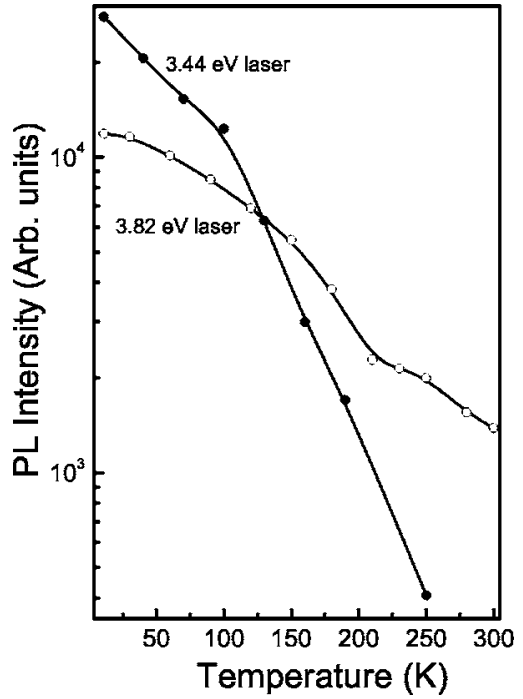

FIG. 5. Different temperature behaviors of the integrated QDs PL intensity of the blue LED excited with laser energies of 3.82 and $3.44 \mathrm{eV}$.

comparison with the absorption spectrum of GaN. This reveals the possibility of a rather accurate determination of the defect location. Furthermore, we do not see any PL signal at this $D$-peak energy region in all the $n$-GaN samples. To deny the possible origin of the $D$ peak from the $P$-GaN capping layer, a reference sample with the same structure but without a $P-G a N$ capping layer was also grown. The PL spectra from the reference sample give the same $D$ peak, of which the integrated intensity follows the same temperature dependence as that in the EL from the LED. We hence strongly believe that this high-energy $D$ peak comes from the GaN barrier or interface in the quantum-well region, which is consistent with the results of the nanometer scale depth-resolved low-energy electron-excited nanoscale-luminescence (LEEN) spectroscopy ${ }^{11}$ which shows that a $3.25-\mathrm{eV}$ localized state can only be observed near the InGaN/GaN interface. The possible physical interpretation of this defect will be shown and discussed by using the transmission electron microscopy (TEM) pictures later.

The effect of the existence of this defect level in the GaN barrier region on transferring carriers into their nearby InGaN quantum dots was reexamined by comparing the different temperature dependences of the integrated PL intensity of QDs excited with laser energies above and below the barrier band gap. We can see in Fig. 5 that both PL intensities show a similar decrease with increase of temperature, as far as it is below $100 \mathrm{~K}$, which is due to the normal thermal quenching process that carriers are thermally annihilated by nonradiative centers. The main difference is that at higher temperatures the decrease of the PL intensity is much faster when it is excited by a 3.44-eV laser than that excited by a 3.82-eV (above the GaN barrier) laser. It seems that in the latter case there is another carrier supply channel (a reservoir of carriers) which is strongly suppressed at low temperatures but thermally activated at higher temperatures. These defrozen carriers will then transfer to the InGaN quantum dots, partially compensating the carrier loss in the thermal quenching process. We attribute this to the release of the carriers captured by the defects in the GaN barrier. 


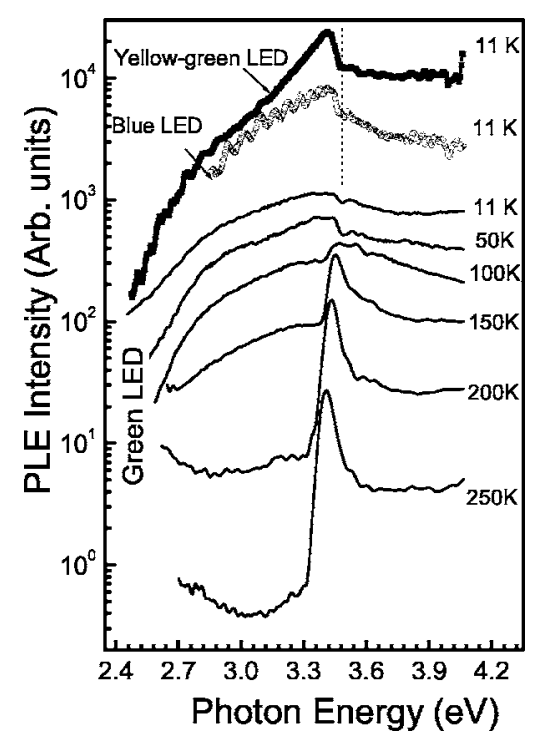

FIG. 6. Temperature-dependent PLE spectra of green LED monitored at QD emission. The PLE spectra of the blue and yellow-green LEDs at $11 \mathrm{~K}$ were also shown as indicated.

The thermally activated carrier transfer process was more carefully examined and clearly demonstrated in the temperature-dependent PLE spectra. The top three curves in Fig. 6 are the PLE spectra of the yellow-green, blue, and green LEDs at $11 \mathrm{~K}$, respectively. We can find that all of them exhibit relatively lower excitation efficiency when the photon energy is higher than the band gap of GaN. When the photon energy is lower than the band gap of GaN, a sharp increase of the excitation efficiency was observed in all the three kinds of LEDs. This shows that the direct photon absorption of InGaN itself induces strong light emission from the QDs. At the band-gap energy of GaN, as marked by the dashed line in Fig. 6, there forms a dip in the PLE spectra, showing the poor transfer efficiency of the optical-generated carriers from the $\mathrm{GaN}$ barriers to the neighboring InGaN QDs. But as the temperature increases, we can find that the contribution from the GaN barrier starts to grow up. At $100 \mathrm{~K}$, the dip in the PLE spectra at the band edge of GaN finally develops into a peak. This implies that at high temperatures the carriers transferred from those generated in the $\mathrm{GaN}$ barriers eventually dominate the PL emission of the InGaN quantum dots. This transition temperature observed around $100 \mathrm{~K}$ is in excellent agreement with those observed in EL and PL experiments.

To reveal the possible physical origin of the $D$-peak-related defects, high-resolution transmission electron microscopy (HRTEM) picture in the active region of the blue LED was taken and presented in Fig. 7. The first feature one can find is that the InGaN potential-well layer is made up of many nanoscaled quantum dots. It is noted that in the enlarged picture, a large number of stacking faults nearby the quantum dots were exposed. The positions of the stacking faults were indicated as arrows in Fig. 7. The enlarged area of a typical stacking fault was shown in the inset. We have counted the number of the stacking faults over the number of QDs as an index of the density of the defects, which is about $43 / 150$. These densely existed defects are so closely located

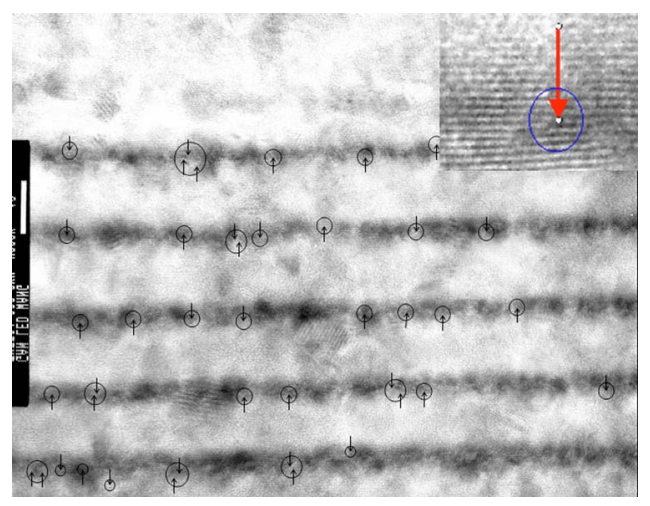

FIG. 7. HRTEM pictures of the active region of a blue LED. The arrows indicate the location of the stacking faults. The enlarged picture of a stacking fault is shown in the insert.

around the QDs that it is not surprising that they can greatly affect the carrier transfer process from the GaN barrier to InGaN QDs. Theoretically, Bandic et al. ${ }^{12}$ have calculated the electronic structure of the GaN stacking faults in the framework of the local empirical pseudopotential theory. The stacking faults in both zinc-blende and wurtzite GaN are predicted to introduce electronic levels within the band gap, with the energy of $0.13 \mathrm{eV}$ above the valence-band top. It was also pointed out that the possible luminescence transition from the conduction band to the stacking fault states would be $3.28 \mathrm{eV}$ for wurtzite $\mathrm{GaN}$. These energies are in good agreement with our observed PL (EL) energy around $3.27 \mathrm{eV}$ and also the thermal activation energy of 0.14-0.15 eV. In spite of the TEM observation, we still do not have evidence to directly correlate the metastable states with the stacking faults. We believe the observed densely existed stacking faults will strongly affect the carrier transfer processes in the quantum-well region and they can explain the experimental observation of the metastable states. However, we still cannot exclude the possible contribution of the donor-acceptor (DA) pairs, which could also show similar emission energy and thermal activation energy. The most possible situation is that the metastable states are the combination of the stacking faults and DA pairs.

\section{CONCLUSIONS}

In conclusion, it was shown that at low temperature the carrier transfer efficiency from the GaN barrier to the InGaN QDs is low due to some excess loss channels associated with defects. But it is found that an efficient carrier transfer process could be thermally activated above $100 \mathrm{~K}$, leading to a good performance of various colored LEDs at high temperatures, despite the existence of high density of defects. These defects thermally release captured carriers which are then transferred into the QDs. They are identified most possibly as stacking faults in the GaN barrier by its energy position, HRTEM pictures, temperature-dependent behavior, and also the comparison with the theoretical predictions. The contribution of DA pairs is another possible origin for the defects observed.

The authors are grateful to the support of Hong Kong RGC Grant No. HKUST 6076/02P. 
${ }^{1}$ M. Sugawara, Phys. Rev. B 51, 10743 (1995).

${ }^{2}$ S. Chichibu, T. Azuhata, T. Sota, and S. Nakamura, Appl. Phys. Lett. 69, 4188 (1996).

${ }^{3}$ S. Chichibu, K. Wada, and S. Nakamura, Appl. Phys. Lett. 71, 2346 (1997).

${ }^{4}$ K. P. O’Donnell, R. W. Martin, and P. G. Middleton, Phys. Rev. Lett. 82, 237 (1999).

${ }^{5}$ A. Hori, D. Yasunaga, A. Satake, and K. Fujiwara, Appl. Phys. Lett. 79, 3724 (2001)

${ }^{6}$ A. Hori, D. Yasunaga, A. Satake, and K. Fujiwara, J. Appl. Phys. 93, 3152 (2003).
${ }^{7}$ A. K. Ghosh and T. Feng, J. Appl. Phys. 49, 5982 (1978).

${ }^{8}$ C. L. Yang, Z. K. Tang, W. K. Ge, J. N. Wang, Z. L. Zhang, and X. Y. Jian, Appl. Phys. Lett. 83, 1737 (2003).

${ }^{9}$ M. O. Manasreh, Phys. Rev. B 53, 16425 (1996).

${ }^{10}$ Diffusion length of the minority carriers in GaN was reported ranging from several nanometers to $200 \mathrm{~nm}$, depending on the defects density in the samples.

${ }^{11}$ T. M. Levin, G. H. Jessen, F. A. Ponce, and L. J. Brillson, J. Vac. Sci. Technol. B 17, 2545 (1999).

${ }^{12}$ Z. Z. Bandic, T. C. McGill, and Z. Ikonic, Phys. Rev. B 56, 3564 (1997). 